\title{
Factors Associated with Medication-Related Burden Quality of Life (MRB-QoL) in Community-Dwelling Adults with Long-Term Conditions: An Exploratory Study
}

This article was published in the following Dove Press journal:

Patient Related Outcome Measures

\author{
Mohammed A Mohammed (D) \\ Rebekah J Moles (iD) ${ }^{2}$ \\ Timothy F Chen (D) ${ }^{2}$ \\ 'University of Auckland, School of \\ Pharmacy, Auckland, New Zealand; ${ }^{2}$ The \\ University of Sydney, School of Pharmacy, \\ Sydney, NSW, Australia
}

Background: The Medication-Related Burden Quality of Life (MRB-QoL) tool has been developed to measure the burden of medications on functioning and wellbeing from a patient perspective. However, predictors of MRB-QoL were not reported in greater detail in the validation study. This study aimed to explore factors associated with MRB-QoL to see whether there is any new information that calls for further research.

Methods: Analysis of data from the MRB-QoL validation study was undertaken. Outcome variables were domains of the MRB-QoL (Routine and Regimen Complexity, Psychological Burden, Functional and Role Limitation, Therapeutic Relationship, and Social Burden). Explanatory variables were patient age; disease-related factors; and medication-related factors, such as number of medications, complexity of medication regimen (measured by the Medication Regimen Complexity Index [MRCI]), and exposure to medications with anticholinergic and sedative effects (measured by the Drug Burden Index [DBI]). Linear regression analyses were used to identify factors associated with the MRB-QoL.

Results: The study included 367 participants (52.1\% male), with a median age of 64 years. In multivariable regression analyses, an increase in the DBI was significantly associated with poorer Psychological wellbeing $(\beta=-0.15, p<0.001)$ and Functional and Role Limitation $(\beta=$ $-1.79, p<0.001)$. Living with three or more medical conditions was significantly associated with poorer Psychological wellbeing $(\beta=-0.21, p<0.001)$. Age was significantly associated with all domains of the MRB-QoL $(\beta=0.28$ to 0.55$)$. Polypharmacy and MRCI were not associated with any of the MRB-QoL domains.

Conclusion: In this sample of community-dwelling adults with multiple medications, the DBI was independently associated with the Psychological Burden and Functional and Role Limitation domains of the MRB-QoL. This study provides preliminary evidence on factors affecting medication-related quality of life outcomes from a patient perspective. Future longitudinal studies, along with further psychometric testing of the MRB-QoL measure, are warranted to better understand predictors of MRB-QoL.

Keywords: patient-reported outcomes, medication-related burden, quality of life, community-dwelling adults

\section{Introduction}

Advances in medicine and public health coupled with improved living standards have significantly contributed to reductions in mortality and increased life expectancy. ${ }^{1-3}$ However, with longevity, most patients live with multiple chronic
Correspondence: Mohammed A Mohammed

The University of Auckland School of Pharmacy, Auckland, New Zealand Tel +6499234458

Email mohammed.mohammed@auckland. ac.nz
Patient Related Outcome Measures 2021:12 55-63

DovePress if in $v$ 
diseases and medications. With appropriate use, medicines improve patients' health outcomes, overall well-being, and functioning. However, there are often negative consequences associated with the long-term use of medicines. These may include adverse events, the burden on day-today life, and the impact on social, financial, psychological, and functional well-being. ${ }^{4-8}$

Health-related quality of life (HRQoL) is an essential treatment goal in pharmacotherapeutic interventions. However, most widely used HRQoL measures are not sensitive enough to detect the changes in HRQoL related to pharmacotherapy. ${ }^{8}$ Thus far, only a few measures, such as the Patient-Reported Outcomes Measure of Pharmaceutical Therapy for Quality of Life (PROMPTQoL), ${ }^{9}$ MedicationRelated Quality of Life (MRQOL), ${ }^{10}$ and MedicationRelated Burden Quality of Life (MRB-QoL), ${ }^{11}$ combining the concept of medication therapy and quality of life have been developed. It has been reported that all of these measures have good initial psychometric properties, yet they have not been widely used in the actual evaluation of the impact of pharmaceutical care (PC) interventions on medication-related quality of life outcomes. Previous studies that have evaluated HRQoL in relation to polypharmacy, ${ }^{12,13}$ regimen complexity, ${ }^{13}$ or pharmacological class of medication ${ }^{14-17}$ have done so using HRQoL measures developed based on chronic disease models, not on drug therapy models. This could be partly because medication-specific measures of quality of life suitable for research purposes have not been developed or are in the early stages of development. However, a large body of evidence shows that although several factors affecting HRQoL have been evaluated using generic or disease-specific measures of HRQoL, the medication-specific burden on patients' well-being ${ }^{18}$ cannot be captured using measures of HRQoL developed based on chronic disease models. ${ }^{19}$ Furthermore, in the validation study of the MRBQoL measure, predictors of medication-related quality of life outcomes were not reported in greater detail. In this study, we used regression analysis to further explore the relationship between the MRB-QoL tool and predictors measured in the MRB-QoL validation study ${ }^{11}$ to see whether there is any new information that calls for further research.

\section{Methods}

\section{Study Population}

The present study focused on secondary analyses of data collected for validation of the MRB-QoL tool ${ }^{11}$ to identify factors associated with the MRB-QoL measure. The
MRB-QoL study was conducted in Sydney, Australia, and data were collected from community-dwelling adults living with chronic conditions and taking multiple medications on a regular basis. ${ }^{11}$ The sample size was estimated based on the ratio of responses per item of the MRB-QoL measure. The link to the MRB-QoL survey was distributed to potential participants. Screening questions were used to allow only eligible participants to complete the questionnaire. Participants were asked to indicate on a five-point Likert scale the extent to which they agreed or disagreed with each statement of the MRBQoL measure, where 1= "strongly agree", 2= "agree", 3= "neither agree nor disagree", 4= "disagree", and $5=$ "strongly disagree". In addition, "prefer not to answer" was included as an alternative option to respect participants' choice of not responding to a given item. A twoweek recall period was used to help participants recall relevant experience associated with medication burden. In total, 367 participants enrolled in the MRB-QoL study. A detailed description of the sample and methods can be found elsewhere. ${ }^{11}$

\section{Study Variables \\ Outcome Measure}

Domains of the MRB-QoL were used as an outcome variable. MRB-QoL is a patient-reported outcome measure of the burden of medicine on functioning and well-being. It has 31 items divided into five domains: Routines and Regimen Complexity (RRC), Psychological Burden (PsyB), Functional and Role Limitation (FRL), Therapeutic Relationship (TR), and Social Burden (SB). All domains of the MRB-QoL have good validity (construct, convergent/divergent, and known groups) and internal consistency reliability (range 0.87-0.95). ${ }^{11}$

\section{Explanatory Variables Medication Factors}

Information about medication use was collected as part of the MRB-QoL tool validation. In the MRB-QoL validation study, participants were asked to provide details of medications, such as name, strength/dose, and number of times a day. The polypharmacy definition of five or more medications ${ }^{13,20,21}$ was used to explore differences among participants based on the number of medications. The use of the anticholinergic and sedative class of medications was quantified using the Drug Burden Index (DBI). ${ }^{15}$ The DBI is a measure of an individual's exposure to medications with anticholinergic and sedative effects 
based on the principle of dose-response and maximum effect. ${ }^{15}$ The burden of complexity of a medication regimen was quantified using the Medication Regimen Complexity Index (MRCI). ${ }^{22}$ The MRCI is the sum of scores of dosage forms, frequency, and additional instructions.

\section{Chronic Disease Factors}

Lists of chronic medical conditions were obtained from patient self-report in the MRB-QoL tool validation. The burden of comorbidities was quantified using the Charlson Comorbidity Index (CCI).

\section{Other Covariates}

Age was the other independent variable included in the analysis.

\section{Statistical Analyses}

Characteristics of the study participants were summarized using descriptive statistics. Normally distributed continuous data are presented as the mean and standard deviation. Non-normally distributed continuous data are presented as the median and interquartile range (IQR), whereas categorical variables are reported using frequencies and percentages. The MRB-QoL subscales comprised non-normally distributed continuous data and, thus, for linear regression analysis the data were transformed using a rank order method. ${ }^{23}$ Tests for linear regression assumptions (linearity, homoscedasticity, normality, and multicollinearity) were conducted to evaluate the suitability of the data. Multicollinearity between each explanatory variable was assessed using tolerance and the variance inflation factor (VIF) (>10 threshold). ${ }^{24}$ Univariate linear regression analysis was conducted to explore the association between the MRB-QoL subscales and each explanatory variable. Variables were retested in multivariable linear regression analyses. Stepwise multiple linear regression analyses were conducted to compare the relative contribution and influence of each explanatory variable on the outcome variables. Thus, medication, disease, and demographic factors were entered in the series of analyses model to explore the variance of MRB-QoL domains explained by each factor. Results are presented using regression coefficients (unstandardized and adjusted) and $p$-values, and the level of statistical significance was determined at a $p$-value $<0.05$. Analyses were conducted using SPSS version 22 .

\section{Results Characteristics of Study Participants}

In total, 367 patients were included in this study $(52.1 \%$ male). The median numbers of chronic medical conditions and prescription medications were 3 and 5, respectively. Most respondents were on five or more medications $(\mathrm{n}=200)$ and living with three or more medical conditions $(n=195)$. The proportion exposed to medication with anticholinergic and sedative effects (ie, DBI $>0$ ) was $52.9 \%$ $(n=148)$. Detailed characteristics of the study participants are presented in Table 1.

Analyses of transformed data showed no evidence of violation of the assumptions of linearity, normality, homoscedasticity, and multicollinearity. Visual inspection of scree plots of standardized residuals supported homoscedasticity and showed no significant outliers. There was no evidence of multicollinearity among independent variables $(\mathrm{VIF}=1$, tolerance $=1)$.

\section{Regression Analyses \\ Routine and Regimen Complexity (RRC) Domain}

The mean score on the RRC domain was $25.2 \pm 10.52$. In linear regression analyses, all variables were significantly associated with RRC scores except for MRCI $(p=0.06)$ (Table 2). The association was stronger for age, DBI, and medical condition. For every unit increase in DBI, the RRC score decreased by $3.04(p<0.01)$. Similarly, for every unit increase in the number of medications and medical conditions, the RRC score decreased by 0.87 $(p<0.01)$ and $1.5(p<0.01)$, respectively. In multivariable regression analyses, age was the only variable significantly associated with the RRC domain (Table 3). In a stepwise

Table I Characteristics of Study Participants ( $N=367)$

\begin{tabular}{|l|l|}
\hline Variables & \\
\hline Age (years), median (IQR) & $64(49-70)$ \\
Male gender, n (\%) & $I 88(5 \mathrm{I} .2)$ \\
Number of medical conditions (IQR) & $3(2-3)$ \\
Number of prescription medications (IQR) & $5(3-7)$ \\
Number of over-the-counter medications (IQR) & $2(I-3)$ \\
$\mathrm{CCl}(\mathrm{IQR})$ & $3(0-4)$ \\
$\mathrm{MRCl}(\mathrm{IQR})$ & $9(7-13)$ \\
Total DBI (IQR) & $0.5(0-0.9)$ \\
$\mathrm{DBI}>0(\mathrm{IQR})$ & $0.9(0.7-\mathrm{I} .6)$ \\
$\mathrm{DBI}=0, \mathrm{n}(\%)$ & $132(47.1)$ \\
$\mathrm{DBI}>0, \mathrm{n}(\%)$ & $148(52.9)$ \\
\hline
\end{tabular}

Abbreviations: $\mathrm{CCl}$, Charlson Comorbidity Index; DBI, Drug Burden Index; IQR, interquartile range; $\mathrm{MRCl}$, Medication Regimen Complexity Index. 
Table 2 Association of Demographic and Clinical Characteristics with MRB-QoL $(\mathrm{N}=367)$

\begin{tabular}{|c|c|c|c|}
\hline Variables & $R^{2}$ & $\begin{array}{c}\beta \text {-Coefficient } \\
\text { (Unstandardized) }\end{array}$ & p-Value \\
\hline \multicolumn{4}{|l|}{$\begin{array}{l}\text { Routine and regimen } \\
\text { complexity (RRC) }\end{array}$} \\
\hline DBI & 0.23 & -3.04 & $<0.01 *$ \\
\hline $\mathrm{CCl}$ & 0.17 & 0.86 & $<0.01 *$ \\
\hline $\mathrm{MRCl}$ & 0.11 & -0.21 & 0.06 \\
\hline Number of medical conditions & 0.21 & -1.54 & $<0.0 I^{*}$ \\
\hline Number of medications & 0.22 & -0.87 & $<0.01 *$ \\
\hline Age & 0.52 & 3.5 & $<0.0 I^{*}$ \\
\hline \multicolumn{4}{|l|}{$\begin{array}{l}\text { Psychological Burden } \\
\text { (PsyB) }\end{array}$} \\
\hline $\mathrm{DBI}$ & 0.26 & -1.96 & $<0.01 *$ \\
\hline $\mathrm{CCl}$ & 0.14 & 0.42 & $0.02 *$ \\
\hline $\mathrm{MRCl}$ & 0.13 & -0.14 & $0.03 *$ \\
\hline Number of medical conditions & 0.23 & -0.97 & $<0.01 *$ \\
\hline Number of medications & 0.14 & -0.32 & $0.01 *$ \\
\hline Age & 0.32 & 0.12 & $<0.01 *$ \\
\hline \multicolumn{4}{|l|}{ Functional and Role } \\
\hline \multicolumn{4}{|l|}{ Limitation (FRL) } \\
\hline DBI & 0.29 & -2.53 & $<0.01 *$ \\
\hline $\mathrm{CCl}$ & 0.19 & 0.64 & $<0.0 I^{*}$ \\
\hline $\mathrm{MRCl}$ & 0.05 & -0.06 & 0.39 \\
\hline Number of medical conditions & 0.18 & -0.87 & $<0.0 I^{*}$ \\
\hline Number of medications & 0.19 & -0.52 & $<0.0 I^{*}$ \\
\hline Age & 0.41 & 0.18 & $<0.0 I^{*}$ \\
\hline \multicolumn{4}{|l|}{$\begin{array}{l}\text { Therapeutic Relationship } \\
\text { (TR) }\end{array}$} \\
\hline $\mathrm{DBI}$ & 0.05 & -0.19 & 0.37 \\
\hline $\mathrm{CCl}$ & 0.18 & 0.25 & $<0.01 *$ \\
\hline $\mathrm{MRCl}$ & 0.03 & 0.02 & 0.61 \\
\hline Number of medical conditions & 0.17 & -0.35 & $<0.01 *$ \\
\hline Number of medications & 0.20 & -0.22 & $<0.0 I^{*}$ \\
\hline Age & 0.43 & 0.08 & $<0.01 *$ \\
\hline \multicolumn{4}{|l|}{ Social Burden (SB) } \\
\hline DBI & 0.19 & -1.02 & $<0.01 *$ \\
\hline $\mathrm{CCl}$ & 0.23 & 0.47 & $<0.0 I^{*}$ \\
\hline $\mathrm{MRCl}$ & 0.02 & -0.02 & 0.74 \\
\hline Number of medical conditions & 0.15 & -0.44 & $<0.01 *$ \\
\hline Number of medications & 0.13 & -0.21 & $0.03^{*}$ \\
\hline Age & 0.50 & 0.14 & $<0.0 I^{*}$ \\
\hline
\end{tabular}

Note: ${ }^{*} p<0.05$.

Abbreviations: $\mathrm{CCl}$, Charlson Comorbidity Index; DBI, Drug Burden Index; MRBQoL, Medication-Related Burden Quality of Life; MRCl, Medication Regimen Complexity Index; $R^{2}$, regression coefficient.

regression analysis in which medication, medical condition, and age variables were sequentially entered into the model, medication-related factors (ie, DBI, MRCI, and number of medications) accounted for $8 \%$ of the variance in the RRC score. Adding medical condition-related factors (ie, $\mathrm{CCI}$ and number of medical conditions) and age to the model increased the total variance to $32 \%$, with age $(\beta=0.37)$ being the only variable significantly associated with RRC score (Table 4).

\section{Psychological Burden (PsyB) Domain}

The mean score on the PsyB domain was $19.7 \pm 6.01$. In linear regression analyses, all variables were significantly associated with the PsyB domain (Table 2). The association was stronger for DBI and medical condition. For every unit increase in DBI, the PsyB domain score decreased by $1.96(p<0.01)$. Similarly, for every unit increase in the number of medical conditions, the PsyB domain score decreased by $0.97 \quad(p<0.01)$. In multiple linear regression analyses, age, DBI, and medical condition were significantly associated with the PsyB domain score (Table 3). After adjusting for medication, medical condition, and age variables, it was found that a unit increase in DBI and number of medical conditions indicated a decrease in the PsyB domain score by 1.5 and 0.21 , respectively. Medication-related factors (ie, DBI, MRCI, and number of medications) accounted for $7 \%$ of the variance in the PsyB domain score. Adding medical condition-related factors (ie, CCI and number of medical conditions) and age to the model increased the variance to $16 \%$ (Table 4 ).

\section{Functional and Role Limitation (FRL) Domain}

The mean score on the FRL domain was $19.3 \pm 6.98$. In linear regression analyses, all variables were significantly associated with the FRL domain score except for MRCI $(p=0.39)$ (Table 2). The association was stronger for DBI and medical condition. For every unit increase in DBI, the FRL score decreased by $2.53(p<0.01)$. Similarly, for every unit increase in the number of medical conditions, the FRL score decreased by $0.87(p<0.01)$. In multivariable regression analyses, DBI and age were the only factors significantly associated with FRL score (Table 3). After adjusting for confounding factors, for every unit increase in DBI and number of medical conditions, the FRL domain score decreased by 0.20 . Medication-related factors (ie, DBI, MRCI, and number of medications) explained $10 \%$ of the variance in FRL score. Addition of medical condition-related factors (ie, CCI and number of medical conditions) and age to the model increased the variability in the FRL domain score to $21 \%$. DBI and age were the two major variables contributing to FRL outcome (Table 4). 
Table 3 Predictors of Medication-Related Burden Quality of Life (MRB-QoL) (N=367)

\begin{tabular}{|c|c|c|c|c|}
\hline \multirow{2}{*}{$\begin{array}{l}\text { Variables } \\
\text { Routine and Regimen Complexity } \\
(\operatorname{RRC}): \mathrm{a} R^{2}=0.32\end{array}$} & \multirow{2}{*}{$\begin{array}{l}\text { Unstandardized } \beta \text { - } \\
\text { Coefficient }\end{array}$} & \multirow{2}{*}{$\begin{array}{l}\text { Standardized } \beta \text { - } \\
\text { Coefficient }\end{array}$} & \multicolumn{2}{|c|}{$95 \% \mathrm{Cl}$} \\
\hline & & & & \\
\hline $\mathrm{DBI}$ & -1.23 & -0.09 & -2.79 & 0.33 \\
\hline $\mathrm{CCl}$ & -0.44 & -0.09 & -0.43 & 0.02 \\
\hline $\mathrm{MRCl}$ & -0.20 & -0.11 & -1.11 & 0.23 \\
\hline Number of medical conditions & -0.15 & -0.02 & -1.29 & 1.00 \\
\hline Number of medications & -0.42 & -0.10 & -1.01 & 0.18 \\
\hline Age & $0.37^{*}$ & $0.55^{*}$ & 0.29 & 0.45 \\
\hline \multicolumn{5}{|c|}{ Psychological Burden (PsyB): $\mathrm{a} R^{2}=0.16$} \\
\hline $\mathrm{DBI}$ & $-1.15^{*}$ & $-0.15^{*}$ & -2.14 & -0.16 \\
\hline $\mathrm{CCl}$ & 0.02 & 0.01 & -0.40 & 0.45 \\
\hline $\mathrm{MRCl}$ & -0.07 & -0.07 & -0.95 & 0.34 \\
\hline Number of medical conditions & $-0.88 *$ & $-0.21 *$ & -1.61 & -1.50 \\
\hline Number of medications & 0.15 & 0.07 & -0.22 & 0.53 \\
\hline Age & $0.11 *$ & $0.28^{*}$ & 4.13 & 0.01 \\
\hline \multicolumn{5}{|c|}{$\begin{array}{l}\text { Functional and Role Limitation } \\
(\mathbf{F R L}): \mathrm{a} R^{2}=0.2 \mathrm{I}\end{array}$} \\
\hline $\mathrm{DBI}$ & $-1.79 *$ & $-0.20^{*}$ & -2.91 & -0.68 \\
\hline $\mathrm{CCl}$ & -0.08 & -0.02 & -0.56 & 0.39 \\
\hline $\mathrm{MRCl}$ & 0.01 & 0.01 & -0.15 & 0.17 \\
\hline Number of medical conditions & -0.12 & -0.03 & -0.94 & 0.69 \\
\hline Number of medications & -0.31 & -0.12 & -0.73 & 0.11 \\
\hline Age & $0.16 *$ & $0.37 *$ & 0.11 & 0.22 \\
\hline \multicolumn{5}{|c|}{ Therapeutic Relationship (TR): $\mathrm{a} R^{2}=\mathbf{0 . 2 0}$} \\
\hline $\mathrm{DBI}$ & 0.22 & 0.05 & -0.26 & 0.69 \\
\hline $\mathrm{CCl}$ & 0.03 & 0.02 & -0.18 & 0.23 \\
\hline $\mathrm{MRCl}$ & 0.004 & 0.008 & -0.06 & 0.07 \\
\hline Number of medical conditions & -0.14 & -0.07 & -0.48 & 0.21 \\
\hline Number of medications & -0.15 & -0.13 & -0.33 & 0.03 \\
\hline Age & $0.08 *$ & $0.4 I^{*}$ & 0.05 & 0.10 \\
\hline \multicolumn{5}{|l|}{ Social Burden (SB): $\mathrm{a} R^{2}=0.26$} \\
\hline $\mathrm{DBI}$ & -0.39 & -0.07 & -1.05 & 0.27 \\
\hline $\mathrm{CCl}$ & -0.02 & -0.01 & -0.30 & 0.26 \\
\hline $\mathrm{MRCl}$ & -0.03 & -0.04 & -0.12 & 0.07 \\
\hline Number of medical conditions & -0.22 & -0.07 & -0.70 & 0.27 \\
\hline Number of medications & -0.03 & -0.02 & -0.28 & 0.22 \\
\hline Age & $0.13^{*}$ & $0.49 *$ & 0.09 & 0.17 \\
\hline
\end{tabular}

Notes: $\beta$, regression coefficient (change in the MRB-QoL domain in relation to the independent variables in the model adjusted by the rest of the covariates). *Indicates variables with significant $(p<0.05)$ association with MRB-QoL domains; bold values denote $R^{2}$.

Abbreviations: $R^{2}$, adjusted regression coefficient; $\mathrm{CCl}$, Charlson Comorbidity Index; $\mathrm{Cl}$, Confidence Interval; $\mathrm{DBI}$, Drug Burden Index; $\mathrm{MRCl}$, Medication Regimen Complexity Index.

\section{Therapeutic Relationship (TR) Domain}

The mean score on the TR domain was $6.5 \pm 2.93$. In linear regression analyses, all variables were significantly associated with TR domain scores except for MRCI $(p=0.61)$ (Table 2). In multivariable regression analysis, there was a significant association between age and TR domain (Table 3). Medication-related factors (ie, DBI,
MRCI, and number of medications) accounted for $4 \%$ of the variance in TR score. After adding medical condition-related factors (ie, CCI and number of medical conditions) and age, the total variance explained by the three factors increased to $19 \%$, with age $(\beta=0.08)$ being the only variable significantly associated with TR outcome (Table 4). 
Table 4 Influence of Demographic and Clinical Characteristics on MRB-QoL (N=367)

\begin{tabular}{|c|c|c|c|c|c|c|c|c|c|}
\hline \multirow{3}{*}{$\begin{array}{l}\text { Variables } \\
\text { Routine and Regimen Complexity (RRC) }\end{array}$} & \multicolumn{3}{|c|}{ Model I } & \multicolumn{3}{|c|}{ Model II } & \multicolumn{3}{|c|}{ Model III } \\
\hline & \multicolumn{3}{|c|}{$\beta(95 \% \mathbf{C l})$} & \multicolumn{3}{|c|}{$\beta(\mathbf{9 5 \%} \mathbf{C l})$} & \multicolumn{3}{|c|}{$\beta(95 \% \mathbf{C l})$} \\
\hline & & & & & & & & & \\
\hline $\mathrm{DBI}$ & $-2.69 *$ & -4.34 & -1.05 & -1.72 & -3.51 & 0.06 & -1.23 & -2.79 & 0.33 \\
\hline $\mathrm{MRCl}$ & 0.01 & -0.23 & 0.24 & -0.07 & -0.32 & 0.19 & -0.20 & -0.43 & 0.02 \\
\hline Number of medications & $-0.76^{*}$ & -0.23 & 0.24 & -0.59 & -1.27 & 0.09 & -0.42 & -1.01 & 0.18 \\
\hline Number of medical conditions & & & & -0.73 & -2.04 & 0.58 & -0.15 & -1.29 & 1.00 \\
\hline $\mathrm{CCl}$ & & & & $0.95 *$ & 0.27 & 1.63 & -0.44 & -1.11 & 0.23 \\
\hline Age & & & & & & & $0.37^{*}$ & 0.29 & 0.45 \\
\hline $\mathrm{a} R^{2}$ & \multicolumn{3}{|l|}{0.08} & \multicolumn{3}{|l|}{0.10} & \multicolumn{3}{|l|}{0.32} \\
\hline \multicolumn{10}{|l|}{ Psychological Burden (PsyB) } \\
\hline DBI & $-1.79 *$ & -2.74 & -0.85 & $-1.29 *$ & -2.31 & 0.28 & $-1.15^{*}$ & -2.14 & -0.16 \\
\hline $\mathrm{MRCl}$ & -0.03 & -0.16 & 0.11 & -0.03 & -0.17 & 0.12 & -0.07 & -0.21 & 0.07 \\
\hline Number of medications & -0.23 & -0.51 & 0.05 & 0.10 & -0.29 & 0.49 & 0.15 & -0.22 & 0.53 \\
\hline Number of medical conditions & & & & $-1.05^{*}$ & -1.79 & -0.30 & $-0.88^{*}$ & $-1.6 \mid$ & -1.50 \\
\hline $\mathrm{CCl}$ & & & & $0.43 *$ & 0.04 & 0.82 & 0.02 & -0.40 & 0.45 \\
\hline Age & & & & & & & $0.1 I^{*}$ & 0.06 & 0.16 \\
\hline $\mathrm{a} R^{2}$ & \multicolumn{3}{|l|}{0.07} & \multicolumn{3}{|l|}{0.10} & \multicolumn{3}{|l|}{0.16} \\
\hline \multicolumn{10}{|l|}{ Functional and Role Limitation (FRL) } \\
\hline $\mathrm{DBI}$ & $-2.55^{*}$ & -3.63 & -1.48 & $-2.01 *$ & -3.19 & -0.84 & $-1.79 *$ & -2.91 & -0.68 \\
\hline $\mathrm{MRCl}$ & 0.11 & -0.5 & 0.26 & 0.07 & -0.10 & 0.23 & 0.01 & -0.15 & 0.17 \\
\hline Number of medications & $-0.47^{*}$ & -0.79 & -0.16 & -0.39 & -0.84 & 0.06 & -0.31 & -0.73 & 0.11 \\
\hline Number of medical conditions & & & & -0.38 & -1.24 & 0.48 & -0.12 & -0.94 & 0.69 \\
\hline $\mathrm{CCl}$ & & & & $0.53^{*}$ & 0.09 & 0.98 & -0.01 & -0.56 & 0.39 \\
\hline Age & & & & & & & $0.16 *$ & 0.11 & 0.22 \\
\hline $\mathrm{a} R^{2}$ & \multicolumn{3}{|l|}{0.10} & \multicolumn{3}{|l|}{0.12} & \multicolumn{3}{|l|}{0.21} \\
\hline \multicolumn{10}{|l|}{ Therapeutic Relationship (TR) } \\
\hline $\mathrm{DBI}$ & -0.21 & -0.68 & 0.25 & 0.11 & -0.39 & 0.62 & 0.22 & -0.26 & 0.69 \\
\hline $\mathrm{MRCl}$ & 0.06 & -0.01 & 0.12 & 0.03 & -0.04 & 0.10 & 0.00 & -0.06 & 0.07 \\
\hline Number of medications & $-0.25^{*}$ & -0.39 & -0.11 & -0.19 & -0.38 & 0.01 & -0.15 & -0.33 & 0.03 \\
\hline Number of medical conditions & & & & -0.26 & -0.63 & 0.11 & -0.14 & -0.48 & 0.21 \\
\hline $\mathrm{CCl}$ & & & & $0.32 *$ & 0.13 & 0.51 & 0.03 & -0.18 & 0.23 \\
\hline Age & & & & & & & $0.08 *$ & 0.05 & 0.10 \\
\hline $\mathrm{a} R^{2}$ & \multicolumn{3}{|l|}{0.04} & \multicolumn{3}{|l|}{0.08} & \multicolumn{3}{|l|}{0.19} \\
\hline \multicolumn{10}{|l|}{ Social Burden (SB) } \\
\hline $\mathrm{DBI}$ & $-1.06 *$ & -1.74 & -0.38 & -0.56 & -1.29 & 0.17 & -0.39 & -1.05 & 0.27 \\
\hline $\mathrm{MRCl}$ & 0.06 & -0.04 & 0.15 & 0.02 & -0.08 & 0.13 & -0.03 & -0.12 & 0.07 \\
\hline Number of medications & $-0.19 *$ & -0.39 & 0.00 & -0.09 & -0.37 & 0.19 & -0.03 & -0.28 & 0.22 \\
\hline Number of medical conditions & & & & -0.43 & -0.96 & 0.11 & -0.22 & -0.70 & 0.27 \\
\hline $\mathrm{CCl}$ & & & & $0.48 *$ & 0.20 & 0.76 & -0.02 & -0.30 & 0.26 \\
\hline Age & & & & & & & $0.13^{*}$ & 0.09 & 0.17 \\
\hline $\mathrm{a} R^{2}$ & 0.04 & & & 0.08 & & & 0.25 & & \\
\hline
\end{tabular}

Notes: Model I: Medication-related factors (number of medications, MRCI, DBI); Model II: adjusted for disease burden factors (CCl, number of medical conditions); Model III: adjusted for demographic factor (age); $R^{2}$, regression coefficient; a $R^{2}$, adjusted $R^{2}$ (ie, the proportion of variance in the MRB-QoL domains explained by independent variables included in the regression models adjusted by the rest of the covariates); bold values denote $\mathrm{a} R^{2} ; \beta$, regression coefficients (the change in the MRB-QoL domain in relation to the independent variables in the model adjusted by the rest of the covariates). *Indicates variables with significant ( $p l<0.05$ ) association with MRB-QoL domains. Abbreviations: CCl, Charlson Comorbidity Index; Cl, confidence Interval; DBI, Drug Burden Index; MRB-QoL, Medication-Related Burden Quality of Life; MRCl, Medication Regimen Complexity Index. 


\section{Social Burden (SB) Domain}

The mean score on the SB domain was 9.9 \pm 4.25 . In linear regression analyses, all variables were significantly associated with SB scores except for MRCI $(p=0.74)$ (Table 2). The association was stronger for DBI, CCI, and number of medical conditions. For every unit increase in DBI and number of medical conditions, the SB score decreased by $1.02(p<0.01)$ and $0.44(p<0.01)$, respectively. In multivariable regression analyses, there was a significant association between age and the SB domain (Table 3). Medication-related factors (ie, DBI, MRCI, and number of medications) accounted for $4 \%$ of the variance in the SB domain score. The total variance in SB domain scores explained by all factors was $25 \%$. Age $(\beta=0.13)$ was the only variable to significantly contribute to SB outcome (Table 4).

\section{Discussion}

This was the first study to explore factors associated with the MRB-QoL outcome measure. The present study showed that DBI was the dominant medication-related factor associated with one or more domains of the MRBQoL. After adjusting for covariates, an increase in DBI was associated with poorer Psychological Burden and Functional and Role Limitation, but not with Routine and Regimen Complexity, Therapeutic Relationship, or Social Burden domain scores. Although number of medicines was significantly associated with all domains of the MRBQoL in univariate analyses, this association was not observed with any of the domains after adjusting for confounding factors. A further analysis by splitting the number of medications, with 5 as a cut-off point for polypharmacy, showed that polypharmacy was not associated with any of the MRB-QoL domains. Similarly, the MRCI was not significantly associated with any of the MRB-QoL domains except for Psychological Burden (in univariate analysis). The lack of association may be due to the lower complexity of the medication regimen, as observed in the calculated MRCI, with a median of 9 .

To the best of our knowledge, no single study has evaluated medicine-specific measure(s) of quality of life in relation to DBI and complexity of the medication regimen to enable a comparison with our findings. Previous cross-sectional and longitudinal studies which used measures of HRQoL developed based on disease models reported significant associations between higher DBI and poorer physical functioning. ${ }^{14,15,17,25}$ Similarly, consistent with our findings, in a previous study, neither MRCI nor polypharmacy was associated with poorer HRQoL outcomes. ${ }^{13}$ However, previous studies ${ }^{12,13}$ evaluating the association between MRCI, polypharmacy, and HRQoL used a non-medicine-specific HRQoL measure, which may not be sensitive enough to capture changes in quality of life pertaining to polypharmacy or complexity of the medication regimen. It is noteworthy that the findings of our study also may not reflect the complete picture of the impact of polypharmacy or complexity of the medication regimen on the patient's quality of life. This may, in part, be due to the fact that the study participants were well-functioning community-dwelling adults with less complex medication regimens, as reflected in the MRCI scores. In addition, there was no access to data to determine evidence regarding the clinical inappropriateness of polypharmacy in the study population. These limitations call for further investigations into the impact of polypharmacy and complexity of medication regimens on quality of life in longitudinal studies and in patients with more complex medication regimens.

In this study, it was found that both CCI and number of medical conditions (as a continuous data) were significantly associated with all subscales of the MRB-QoL (in univariate analysis). However, after adjusting for age and medication-related factors, CCI was not significantly associated with all subscales of the MRB-QoL, whereas the number of medical conditions was associated only with the Psychological Burden domain. The impact of concurrent multiple chronic conditions on poorer HRQoL outcome is well established, with evidence gained from research using non-MRB-QoL measures. ${ }^{26}$ In our study, the lack of association between the number of medical conditions and other domains of the MRB-QoL, such as Functional and Role Limitation, could be explained by the lower severity of disease, as participants were well-functioning community-dwelling adults. However, this study did not explore detailed information regarding the types of medical condition that were significantly associated with poorer psychological well-being. It may be important to investigate whether the association between the number and types of medical conditions and MRB-QoL is driven by the number of medications prescribed for a condition or by other factors.

Another finding in our study was that age was significantly associated with the scores on all MRB-QoL domains. The multivariable analyses results showed that medicationrelated quality of life tends to increase with increasing age. 
While this could be due to a range of reasons, such as being on less complex medication regimens which can be easily managed by patients, participants were well-functioning community-dwelling adults and had low disease complexity; therefore, the overall impact of the disease on their health and well-being may be minimal. Despite this, the direction of the association between age and MRB-QoL domains needs further investigation. Contrary to our findings, an increase in age was associated with a decrease in HRQoL outcomes in a previous study evaluating the impact of chronic disease on HRQoL. ${ }^{27}$

The key strength of this study is the use of a validated medicine-specific measure of medication burden on quality of life. Our findings provide preliminary evidence regarding factors associated with MRB-QoL and highlight the importance of further exploring potential factors affecting medication-related quality of life outcomes in patients with multiple morbidities and multiple medications. This may help clinicians to tailor pharmacotherapeutic interventions and other management strategies to prevent or minimize factors contributing to poorer medication-related quality of life outcomes. Despite this, our study is not without some limitations. Associations between MRBQoL and sociodemographic factors such as income level, and education/health literacy were not evaluated in this study. Moreover, owing to the cross-sectional nature of the data, it was not possible to infer causal relationships between poorer MRB-QoL outcomes and explanatory variables. Data were obtained from the MRB-QoL validation survey of well-functioning community-dwelling adults and, thus, results may be different for hospitalized patients and for patients with severe conditions or on clinically inappropriate polypharmacy. Furthermore, there may be a possibility of information bias owing to the selfreported nature of data on medication and medical conditions, which we did not confirm through reviewing medical records and medication charts. Although we have included potential factors that may influence medicationrelated quality of life outcomes, the models explained only $16-32 \%$ of the variability in MRB-QoL outcomes. This could be due to the cross-sectional nature of the data or there could be other unexplored factors potentially explaining the remaining variability in MRB-QoL outcomes. Moreover, the types and severity of medical conditions, and the clinical appropriateness of medications were not evaluated, and this may have affected the association between explanatory variables and MRB-QoL domains. Future investigations could therefore include exploring the medication-related burden in different populations, such as patients recruited from hospitals and nursing homes, and those with different medical conditions, more complex medication regimens, and problematic polypharmacy.

\section{Conclusion}

The findings of this study provide preliminary evidence on potential factors affecting medication-related quality of life outcomes. DBI was the dominant medication-related factor, which was significantly associated with poorer scores on the Psychological Burden and Functional and Role Limitation domains of the MRB-QoL. An increase in the number of medical conditions was associated with poorer psychological well-being. Neither polypharmacy nor MRCI was associated with any of the MRB-QoL domains. Future longitudinal studies along with further psychometric testing of the MRB-QoL measure are warranted to better understand predictors of medicationrelated quality of life outcomes.

\section{Ethical Approval}

Ethical approval was obtained from the Human Ethics Committee, the University of Sydney (project number 2016/654). Prior to completion of the survey, all participants received a participant information sheet (PIS) and consent form (CF) containing detailed information about the study to help them make an informed decision. As approved by the ethics committee, informed consent was conferred through the return of completed questionnaires. The study was conducted in accordance with the Declaration of Helsinki.

\section{Acknowledgments}

The authors thank the participants of the study for their time.

\section{Funding}

No specific funding was received for this work.

\section{Disclosure}

The authors declare no competing interest in this work.

\section{References}

1. Preston SH. Mortality Patterns in National Populations: With Special Reference to Recorded Causes of Death. New York: Academic. Press; 1976. 
2. Moser K, Shkolnikov V, Leon DA. World mortality 1950-2000: divergence replaces convergence from the late 1980s. Bull World Health Organ. 2005;83:202-209.

3. McMichael AJ, McKee M, Shkolnikov V, Valkonen T. Mortality trends and setbacks: global convergence or divergence? Lancet. 2004;363:1155-1159. doi:10.1016/S0140-6736(04)15902-3

4. Aston J, Wilson KA, Terry DRP. The treatment-related experiences of parents, children and young people with regular prescribed medication. Int $J$ Clin Pharm. 2019;41:113-121. doi:10.1007/ s11096-018-0756-Z

5. Zhang S, Meng L, Qiu F, Yang JD, Sun S. Medication-related risk factors associated with health-related quality of life among community-dwelling elderly in China. Patient Prefer Adherence. 2018;12:529-537. doi:10.2147/PPA.S156713

6. van der Laan DM, Elders PJM, Boons CCLM, et al. The impact of cardiovascular medication use on patients' daily lives: a crosssectional study. Int J Clin Pharm. 2018;40:412-420. doi:10.1007/ s11096-018-0601-4

7. Krska J, Katusiime B, Corlett SA. Patient experiences of the burden of using medicines for long-term conditions and factors affecting burden: a cross-sectional survey. Health Soc Care Community. 2018;26:946-959. doi:10.1111/hsc.12624

8. Mohammed MA, Moles RJ, Chen TF. Impact of pharmaceutical care interventions on health-related quality-of-life outcomes: a systematic review and meta-analysis. Ann Pharmacother. 2016;50(10):862-881. doi:10.1177/1060028016656016

9. Sakthong P, Suksanga P, Sakulbumrungsil R, Winit-Watjana W. Development of patient-reported outcomes measure of pharmaceutical therapy for Quality of Life (PROMPT-QoL): a novel instrument for medication management. Res Social Adm Pharm. 2015;11 (3):315-338. doi:10.1016/j.sapharm.2014.10.002

10. Tseng HM, Lee CH, Chen YJ, Hsu HH, Huang LY, Huang JL. Developing a measure of medication-related quality of life for people with polypharmacy. Qual Life Res. 2016;25(5):1295-1302. doi:10.1007/s11136-015-1177-2

11. Mohammed MA, Moles RJ, Kouladjian L, Hilmer S, Chen TF. Development and validation of patient reported outcome measure of medication burden: the medication-related burden for quality of life (MRB-QoL) tool. BMJ Open. 2018;8:e018880. doi:10.1136/bmjopen-2017-018880

12. Montiel-Luque A, Nunez-Montenegro AJ, Martin-Aurioles E, CancaSanchez JC, Toro-Toro MC, Gonzalez-Correa JA. Medication-related factors associated with health-related quality of life in patients older than 65 years with polypharmacy. PLoS One. 2017;12(2):e0171320. doi: 10.1371 journal.pone. 0171320

13. Lalic S, Jamsen KM, Wimmer BC, et al. Polypharmacy and medication regimen complexity as factors associated with staff informant rated quality of life in residents of aged care facilities: a cross-sectional study. Eur J Clin Pharmacol. 2016;72 (9):1117-1124. doi:10.1007/s00228-016-2075-4

14. Hilmer SN, Mager DE, Simonsick EM, et al. Drug burden index score and functional decline in older people. Am J Med. 2009;122 (12):1142-9.e1-2. doi:10.1016/j.amjmed.2009.02.021
15. Hilmer SN, Mager DE, Simonsick EM, et al. A drug burden index to define the functional burden of medications in older people. Arch Intern Med. 2007;167(8):781-787. doi:10.1001/archinte.167.8.781

16. Lowry E, Woodman RJ, Soiza RL, Hilmer SN, Mangoni AA. Drug burden index, physical function, and adverse outcomes in older hospitalized patients. J Clin Pharmacol. 2012;52(10):1584-1591. doi:10.1177/0091270011421489

17. Gnjidic D, Cumming RG, Le Couteur DG, et al. Drug Burden Index and physical function in older Australian men. Br J Clin Pharmacol. 2009;68(1):97-105. doi:10.1111/j.1365-2125.2009.03411.x

18. Mohammed MA, Moles RJ, Chen TF. Medication-related burden and patients' lived experience with medicine: a systematic review and metasynthesis of qualitative studies. BMJ Open. 2016;6:2. doi:10.1136/bmjopen-2015-010035

19. Mohammed MA, Moles RJ, Chen TF. Pharmaceutical care and health related quality of life outcomes over the past 25 years: have we measured dimensions that really matter? Int $J$ Clin Pharm. 2018;40:3-14. doi:10.1007/s11096-017-0582-8

20. Gnjidic D, Hilmer SN, Blyth FM, et al. Polypharmacy cutoff and outcomes: five or more medicines were used to identify community-dwelling older men at risk of different adverse outcomes. J Clin Epidemiol. 2012;65(9):989-995. doi:10.1016/j. jclinepi.2012.02.018

21. Turner JP, Jamsen KM, Shakib S, Singhal N, Prowse R, Bell JS. Polypharmacy cut-points in older people with cancer: how many medications are too many? Support Care Cancer. 2016;24 (4):1831-1840. doi:10.1007/s00520-015-2970-8

22. George J, Phun YT, Bailey MJ, Kong DC, Stewart K. Development and validation of the medication regimen complexity index. Ann Pharmacother. 2004;38(9):1369-1376. doi:10.1345/aph.1D479

23. Templeton GF. A two-step approach for transforming continuous variables to normal: implications and recommendations for IS research. Communications Assoc Information Systems. 2011;28 (4):41-58. doi:10.17705/1CAIS.02804

24. O'brien RM. A caution regarding rules of thumb for variance inflation factors. Qual Quant. 2007;41(5):673-690. doi:10.1007/s11135006-9018-6

25. Gnjidic D, Bell JS, Hilmer SN, Lonnroos E, Sulkava R, Hartikainen S. Drug Burden Index associated with function in community-dwelling older people in Finland: a cross-sectional study. Ann Med. 2012;44(5):458-467. doi:10.3109/ 07853890.2011.573499

26. Gallegos-Carrillo K, Garcia-Pena C, Mudgal J, Romero X, DuranArenas L, Salmeron J. Role of depressive symptoms and comorbid chronic disease on health-related quality of life among community-dwelling older adults. J Psychosom Res. 2009;66 (2):127-135. doi:10.1016/j.jpsychores.2008.07.007

27. Zyoud SH, Daraghmeh DN, Mezyed DO, et al. Factors affecting quality of life in patients on haemodialysis: a cross-sectional study from Palestine. BMC Nephrol. 2016;17(1):44. doi:10.1186/s12882016-0257-z
Patient Related Outcome Measures

\section{Publish your work in this journal}

Patient Related Outcome Measures is an international, peer-reviewed, open access journal focusing on treatment outcomes specifically relevant to patients. All aspects of patient care are addressed within the journal and practitioners from all disciplines are invited to submit their work as well as healthcare researchers and patient support groups.
Dovepress

The manuscript management system is completely online and includes a very quick and fair peer-review system. Visit http://www. dovepress.com/testimonials.php to read real quotes from published authors. 